\title{
Dinámica de la flora arvense en forestaciones de terrenos agrícolas
}

\section{Dynamics of weeds in forestation of agricultural land}

Monreal, J.A.; Copete, M.A.; Botella, O.; Herranz, J.Mª Ferrandis, P.

*Departamento de Producción Vegetal y Tecnología Agraria.

Escuela Técnica Superior de Ingenieros Agrónomos y de Montes.

Universidad de Castilla-La Mancha, Campus Universitario s/n, 02071 Albacete

Autor para correspondencia: jose.monreal@uclm.es 


\title{
Resumen
}

En los últimos 20 años se han forestado en Castilla La-Mancha cerca de 120.000 ha de terrenos agrícolas, correspondiendo unas 30.000 ha a la provincia de Albacete. En las forestaciones de terrenos agrícolas se produce, durante los primeros años, una fuerte competencia entre la flora arvense y las plantas forestales. Sin embargo, con el paso de los años la cobertura de la vegetación forestal puede acabar reduciendo la diversidad vegetal del sotobosque. En este trabajo se ha estudiado la evolución de la flora arvense con la edad de las forestaciones para conocer la dinámica de colonización de herbáceas y leñosas, así como los cambios en la comunidad a partir de la transformación de un terreno agrícola a forestal. Para ello, se han seleccionado 36 forestaciones pertenecientes a tres grupos de edad: de 1 a 7 años; de 8 a 14 años y de 15 a 21 años. En todas ellas se han identificado las especies vegetales presentes y estimado su cobertura, y se ha calculado la riqueza específica y el índice de diversidad de Shannon-Wiener. Los resultados indican que, a partir de los 7 años, el número de especies de flora arvense presentes en las forestaciones se reduce en un 25\%; y la cobertura de estas especies se reduce en más de un $70 \%$ a partir de los 14 años. El estudio nos permite concluir que, si no se realizan tareas selvícolas en la forestación, el reclutamiento de especies herbáceas y leñosas tendrá grandes dificultades.

Palabras clave: diversidad, forestaciones en terrenos agrícolas, sucesión en comunidades arvenses.

\begin{abstract}
In the last 20 years, about 120,000 ha of agricultural land have been reforested in Castilla-La Mancha. About 30,000 ha in the province of Albacete. The afforestation of agricultural land can produce intense competition between weeds and forest species during the first years, however, as the forest vegetation grows the vegetative competition diminishes due to plant diversity reduction. In this work, we have studied the evolution of weeds with the age of afforestation to assess the dynamics of herbaceous and woody colonization. To find out the changes in the vegetal community from arable land to forest, we selected 36 afforestation sites from three age groups: from 1 to 7 years, 8 to 14 years and 15 to 21 years. In all of them, the present plant species have been identified and its coverage, the specific wealth and Shannon-Wiener index to know the biodiversity for each of forestation have been estimated. The results show that from the 7 years the number of species of weed in forestation is reduced to $25 \%$. The weed cover decreases by more than $70 \%$ from 14 years. The study allows us to conclude that, if silvicultural tasks are not carried out after afforestation, the recruitment of herbaceous and woody forest species will have great difficulties.
\end{abstract}

Keywords: diversity, forestation on agricultural land, succession weed communities. 


\section{Introducción}

Con la reforma de la Política Agraria Común (PAC) en 1992, se desarrolló en España un régimen de ayudas que ha fomentado las inversiones forestales en explotaciones agrarias. Entre 1994 y 2013 se han forestado más de 700.000 ha de terrenos agrícolas abandonados, de las cuales 120.000 ha corresponden a Castilla-La Mancha (Fig. 1) y cerca de 30.000 ha a la provincia de Albacete (Carmona, 2008; MAPAMA, 2017). Las forestaciones se han realizado con especies arbóreas y arbustivas, predominando pinos y encinas entre las primeras y retama y coscoja entre las segundas. En estos proyectos, la Junta de Comunidades de Castilla-La Mancha aportó ayudas al establecimiento de plantaciones y a los cuidados posteriores. Algunas de estas tareas deben ir dirigidas a mantener la forestación, y entre ellas se incluye el control de la flora arvense.

\section{Superficie forestada (ha)}

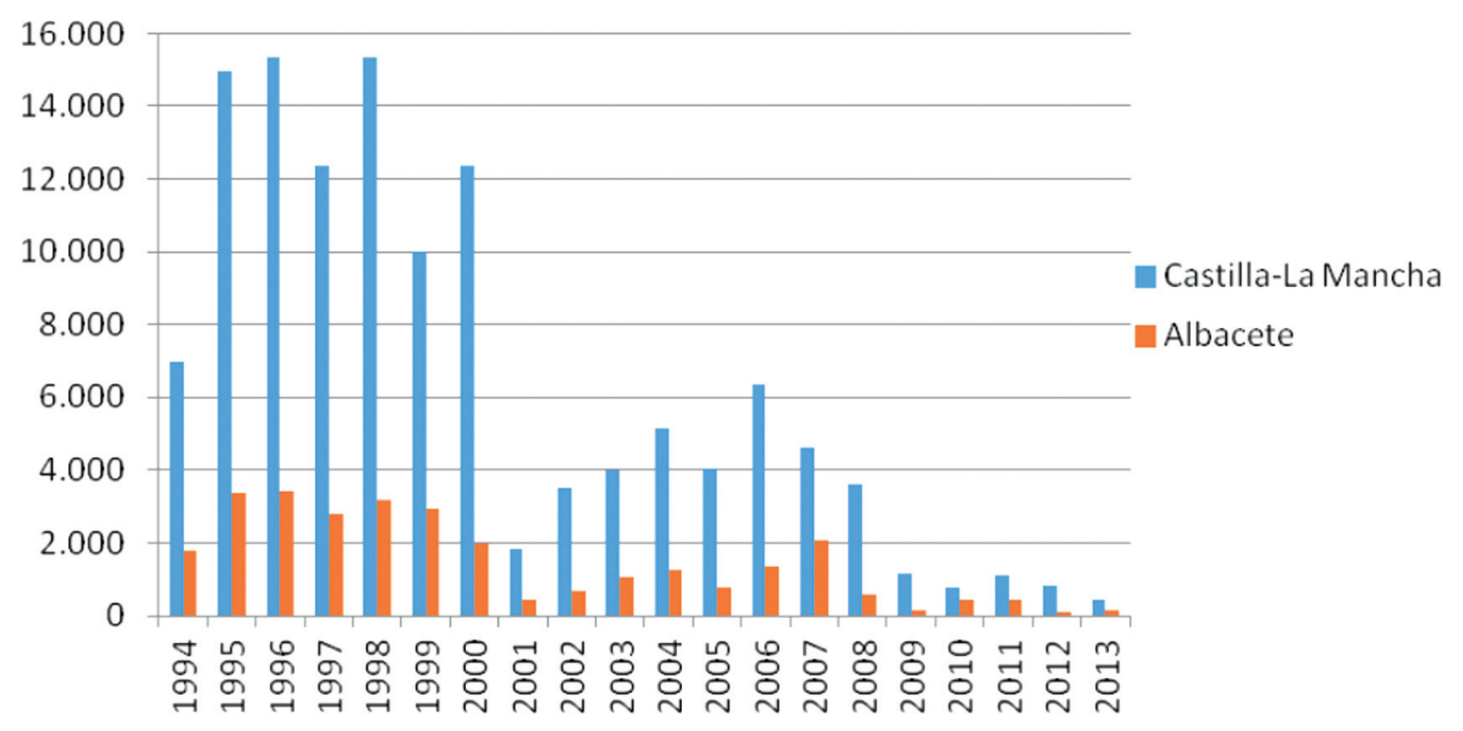

Figura 1. Superficies forestadas en Albacete y Castilla-La Mancha durante los años 1994-2013 (Elaboración propia con datos de la Junta de Comunidades de Castilla-La Mancha).

En la zona de estudio (La Mancha-Centro) con clima seco y semiárido, las forestaciones en terrenos agrícolas abandonados pueden verse notablemente afectadas, durante los primeros años, por la competencia de la flora arvense, tanto por las escasas precipitaciones como por las propias características de los suelos agrícolas, carentes de inóculos micorrícicos y con un gran número de propágulos de malas hierbas (Orozco y Monreal, 1997). La vegetación herbácea tiene una gran capacidad para competir por el agua y los nutrientes cuando éstos escasean en el suelo, disminuyendo el crecimiento de las forestaciones e incluso provocando la mortan- 
dad de las plantas (Van Lerberghe and Balleux, 2001). Es importante destacar también que los terrenos que han estado sometidos a una actividad agrícola prolongada, tienen dificultades para el arraigo de las plantas forestales, debido fundamentalmente a la alteración de los factores físicos, químicos y biológicos generada por dicha actividad (Díaz, 1997).

En esta situación, resulta crucial el control de malas hierbas para la consolidación de las forestaciones. Pero para llevar a cabo un control eficaz de las malas hierbas, es necesario tener un conocimiento previo de la dinámica de la flora arvense a lo largo del tiempo, desde el inicio de la forestación y durante los primeros años hasta que las plantas recién instaladas superan dicha competencia. El estudio de la evolución de la flora arvense con la edad de las forestaciones nos aporta información sobre la dinámica de colonización de herbáceas y leñosas, así como sobre los cambios en la comunidad de flora arvense a partir de la transformación de un terreno agrícola a forestal.

El abandono de tierras agrícolas no es un problema solo en España, en las últimas décadas se han forestado terrenos agrícolas abandonados en diversas partes del mundo, tanto en nuestro continente (Portugal, Francia, etc.), como en otros, Australia, América y África, existiendo diferentes trabajos que muestran los resultados, pero también las inquietudes sobre el futuro de estas forestaciones en cuanto a gestión y biodiversidad (Lemieux and Delisle, 1998; Chapman and Chapman, 1999; Brockerhoff et al., 2008; Kushla, 2009; Pires and Xavier, 2010; Bremer and Farley, 2010).

La mayoría de los investigadores coincide en que los suelos abandonados definitivamente por la agricultura evolucionan de forma diferente según las condiciones climáticas, situación fisiográfica y acción antrópica, con tendencia general hacia la reforestación, siendo un fenómeno muy lento. A veces esta sucesión progresiva puede ser incluso nula, cuando el grado de degradación es muy elevado y las condiciones son poco favorables. En estas ocasiones es necesario intervenir (Francis, 1990). Existen diferentes estudios sobre la evolución natural de los terrenos agrícolas abandonados y su colonización por la vegetación forestal (Francis, 1990; Ruiz De La Torre, 1990a; Sánchez, 1995; Boccanelli, 1999), coincidiendo todos ellos en que esta restauración que se da de forma natural es muy lenta, y mucho más difícil en climas adversos.

Si bien la mayoría de los técnicos e investigadores están de acuerdo en los beneficios que producen las forestaciones, muchos de ellos destacan que dichas forestaciones no han cumplido el verdadero objetivo que pretendían (Montoya, 1997). Hay que recordar que forman parte de la Política Agrícola Comunitaria (PAC) y que uno de sus objetivos era el abandono de tierras y su transformación a terrenos forestales, mientras que muchas de las forestaciones se han realizado en eriales y pastizales con clara vocación forestal, e incluso en algunos casos ya ocupadas por vegetación arbustiva (MAPA, 2006). En este sentido, otros autores señalan que, en algunos suelos degradados, con características edáficas y climáticas muy difíciles, los matorrales deberían ser conservados (Orozco y López, 1993). Según Montiel y Galiana (2004), aunque la forestación de tierras agrícolas ha favorecido en algunos 
casos la restauración de paisajes forestales degradados (zonas con riesgo de erosión, tierras marginales agrarias, terrazas, etc.), también ha contribuido a la homogeneización de dicho paisaje cuando ha afectado a parcelas enclavadas en superficies forestales que desempeñaban una función ecológica y paisajística fundamental (retamares, atochares, etc.) como elemento de diversificación, complementariedad y contraste.

Destaca también la incertidumbre que plantean algunos investigadores sobre la mejora de la biodiversidad, dado que en muchas forestaciones no se han incluido nada más que una o dos especies arbóreas y, en el mejor de los casos, alguna especie arbustiva, habiéndose realizado la mayoría de ellas con altas densidades (Bremer and Farley, 2010), lo que dificulta la colonización de la flora silvestre del entorno. Según Ruiz De La Torre (1993b) la densidad de plantación no debería exceder de 1000 plantas/ha (un marco de 3 x 3,5 m), dado que en el futuro y desde el punto de vista económico parece inviable la eliminación de los pies sobrantes. Para este mismo autor es muy importante tener en cuenta el temperamento de la especie que se va a instalar, dado que uno de los principales factores que pueden hacer fracasar una plantación en suelos desnudos es la insolación directa. Otros autores también indican que una de las dificultades para conseguir que estas forestaciones alcancen una mayor diversidad será, en el futuro, la falta de gestión forestal (Brockerhoff et al., 2008).

En relación con la diversidad, si se consideran los niveles evolutivos de las comunidades en las que participan especies vegetales, para las forestaciones de terrenos agrícolas abandonados habría que buscar aquellas especies que puedan adelantar etapas de sustitución y, por tanto, alcanzar pasos intermedios más evolucionadas, como es el caso de los pinos en este tipo de situaciones semiáridas (Ruiz De La Torre, 1993b). Gil y Prada (1993) también coinciden en que los pinos son especies adecuadas para repoblar terrenos pobres que van a padecer prolongadas sequías y temperaturas extremas.

Es importante indicar, además, desde el punto de vista del manejo futuro de plagas, que si aumenta la biodiversidad se puede favorecer la salud de estas forestaciones, dado que hay una mayor abundancia y diversidad de enemigos naturales en aquellos ecosistemas que son más diversos en especies vegetales. Esto se basa en que en un entorno complejo existe mayor disponibilidad de alimento suplementario suministrado por plantas, así como una mayor diversidad de presas, huéspedes y microhábitats. Por otra parte, una forestación en un terreno agrícola no se convierte automáticamente en una excelente masa forestal, y no aporta siempre los resultados esperados. Para ello es necesario que existan unas condiciones favorables, medios económicos, un seguimiento regular y ciertos conocimientos técnicos, de los que no siempre va a disponer el propietario (Montoya, 1997; Monsalve, 1997).

El objetivo de este trabajo fue conocer la dinámica de la flora arvense con la edad de las forestaciones y evaluar el efecto de la distancia de las forestaciones a otras zonas forestales sobre la diversidad vegetal. 


\section{Material y métodos}

\subsection{Localización de la zona de estudio}

Para la localización de la zona de estudio se solicitó en el año 2013, a la Dirección Provincial de Agricultura, Medio Ambiente y Desarrollo Rural en Albacete, una relación de las forestaciones realizadas en el marco de las ayudas comunitarias a las forestaciones de tierras agrícolas. Con la ayuda de los Técnicos y de los Agentes Medioambientales de la mencionada Dirección Provincial, se seleccionaron y visitaron 36 forestaciones, entre las cuales deberían estar representados homogéneamente todos los rangos de edad.

Todas las forestaciones seleccionadas quedaron incluidas en las hojas 6-8 y 78 del Mapa Forestal de España a escala 1:200.000 (Ruiz de la Torre, 1990b y 1993a) y, lo más importante, todas se seleccionaron de modo que pertenecieran al mismo sector biogeográfico para evitar distorsiones en los resultados. En concreto, dentro de la subprovincia Castellana, el sector elegido ha sido el Manchego, por ser el más representado en la provincia (Sánchez et al., 1997). Las forestaciones están ubicadas en parcelas procedentes de antiguos cultivos agrícolas o terrenos marginales de municipios de la provincia de Albacete, en las siguientes comarcas agrarias: Sierra de Alcaraz (San Pedro), Mancha (Albacete y La Roda), Manchuela (Abengibre y Madrigueras) y Corredor de Almansa (Chinchilla de Montearagón y Bonete).

\subsection{Diseño experimental}

En la primavera de 2014 se visitaron las 36 forestaciones seleccionadas, y se agruparon por edades, dividiéndose en tres grupos de similar tamaño (12 forestaciones por grupo), de 1 a 7 años, de 8 a 14 años y de 15 a 21 años (Fig. 2). Para la selección de las parcelas, además de la clasificación por edades, también se tuvo en cuenta la accesibilidad para facilitar la toma de datos.

Para caracterizar las forestaciones se registraron los siguientes datos: número de especies utilizadas y porcentaje de representación de cada una de ellas, marco de plantación, diámetro de copa de las diferentes especies, cultivo anterior a la forestación, labores realizadas y presencia de herbáceas y leñosas. Para la estimación del diámetro de copa se utilizaba la media del diámetro en cruz, medido con una cinta métrica. Esta variable se midió en 10 plantas de cada una de las especies utilizadas en la forestación, que junto con el porcentaje de cada especie y la densidad de plantación permitió calcular posteriormente la cobertura.

Para el estudio de la flora arvense, durante el mes de mayo de 2016, en cada forestación se marcaron tres parcelas de $3 \times 3 \mathrm{~m}$, una cerca del borde (A), y las otras dos hacia el interior a 25 (B) y 50 (C) metros respectivamente, con el fin de detectar el posible efecto borde relacionado con un mayor número de especies arvenses y su facilidad de colonización. En cada extremo de las parcelas marcadas se colocaba un jalón y se iban recolectando y anotando todas las especies presentes, esti- 


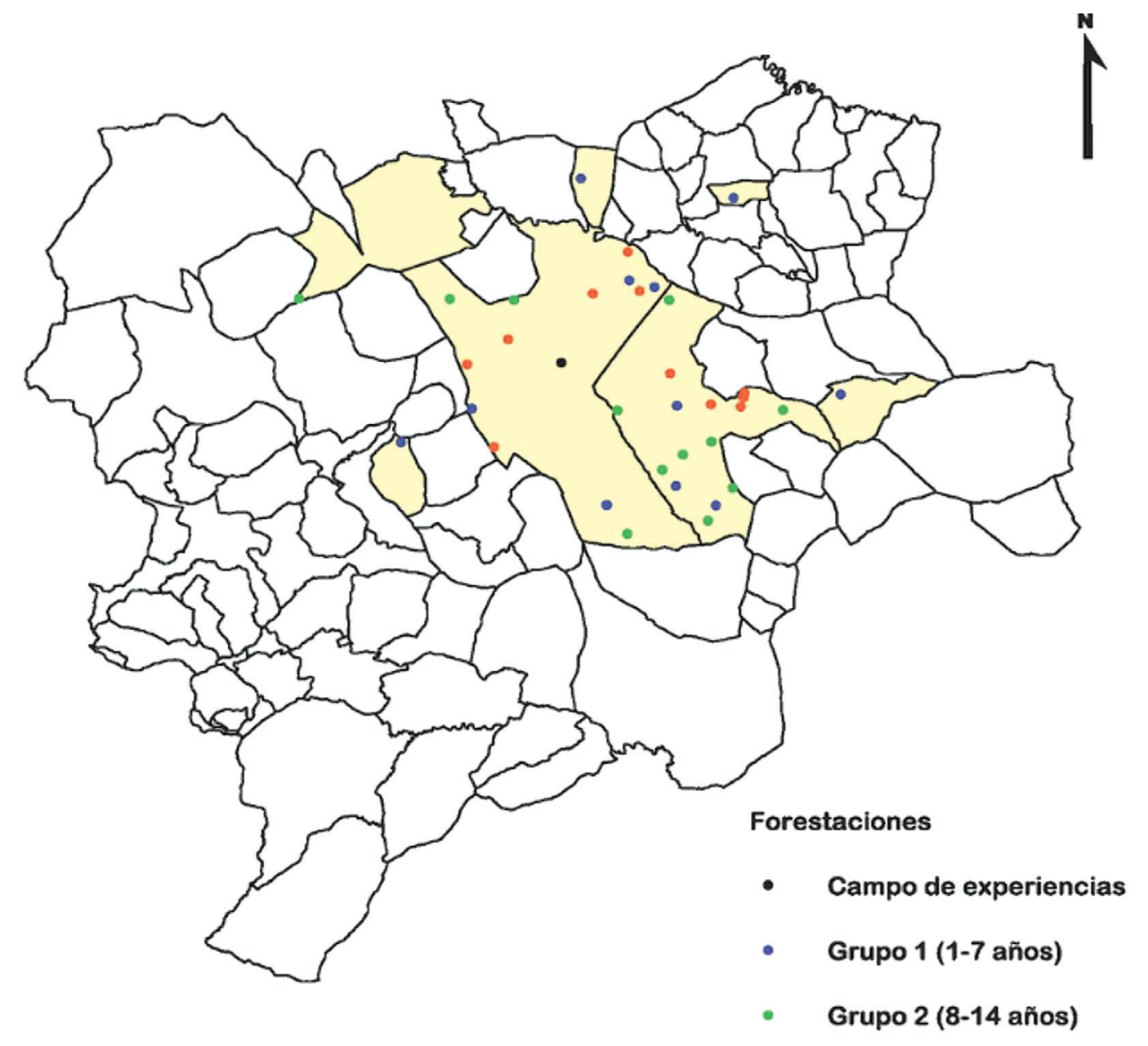

Figura 2. Mapa de localización de las forestaciones estudiadas. Elaboración propia.

mando para cada una su porcentaje de cobertura. En el caso de no poder identificar una especie se recogía y asignaba un número, para después en el laboratorio de la ETSIAM proceder a su identificación.

\subsection{Cuantificación de la diversidad}

Para comprobar en qué momento del intervalo de edades medido en las forestaciones se produce la mayor diversidad de flora arvense, se calcularon diversos índices basados en cuantificar el número de especies presentes o en el estudio de la estructura de la comunidad. En todas las parcelas marcadas (A, B y C) en las distintas forestaciones se calcularon dos siguientes índices: 
1. Riqueza específica (S): número total de especies de un lugar determinado.

2. Índice de diversidad específica de Shannon-Wiener $\left(H^{\prime}\right): H^{\prime}=-\Sigma_{p_{i}} * \ln p_{i}$ siendo $p_{i}$ : proporción de individuos de la especie i respecto al total de individuos (es decir la abundancia relativa de la especie, $p_{i}=n_{i} / N$; $n_{i}$ : número de individuos de la especie i; y $N$ : número total de individuos de todas las especies. Este índice tiene en cuenta la cantidad de especies presentes en el área de estudio (riqueza de especies), y la cantidad relativa de individuos de cada una de esas especies (abundancia) (Escolástico et al., 2006). Varía entre 1 y 5 , considerándose un valor alto de diversidad a partir de 2,5.

\subsection{Cálculo de la distancia a masas forestales}

Para evaluar el efecto de la distancia a masas forestales sobre la diversidad de flora arvense en las forestaciones, se calculó la distancia existente entre estas y la masa forestal más cercana utilizando el programa informático QGIS-2.6.1. Brighton. Concretamente se utilizó la herramienta buffer alrededor de cada forestación para analizar su posible intersección con las masas forestales (arbóreas y arbustivas) más próximas. Cuando ésta fue detectada, se midió la distancia desde el centro de la forestación al punto más cercano de la masa forestal intersectada (Fig. 3).

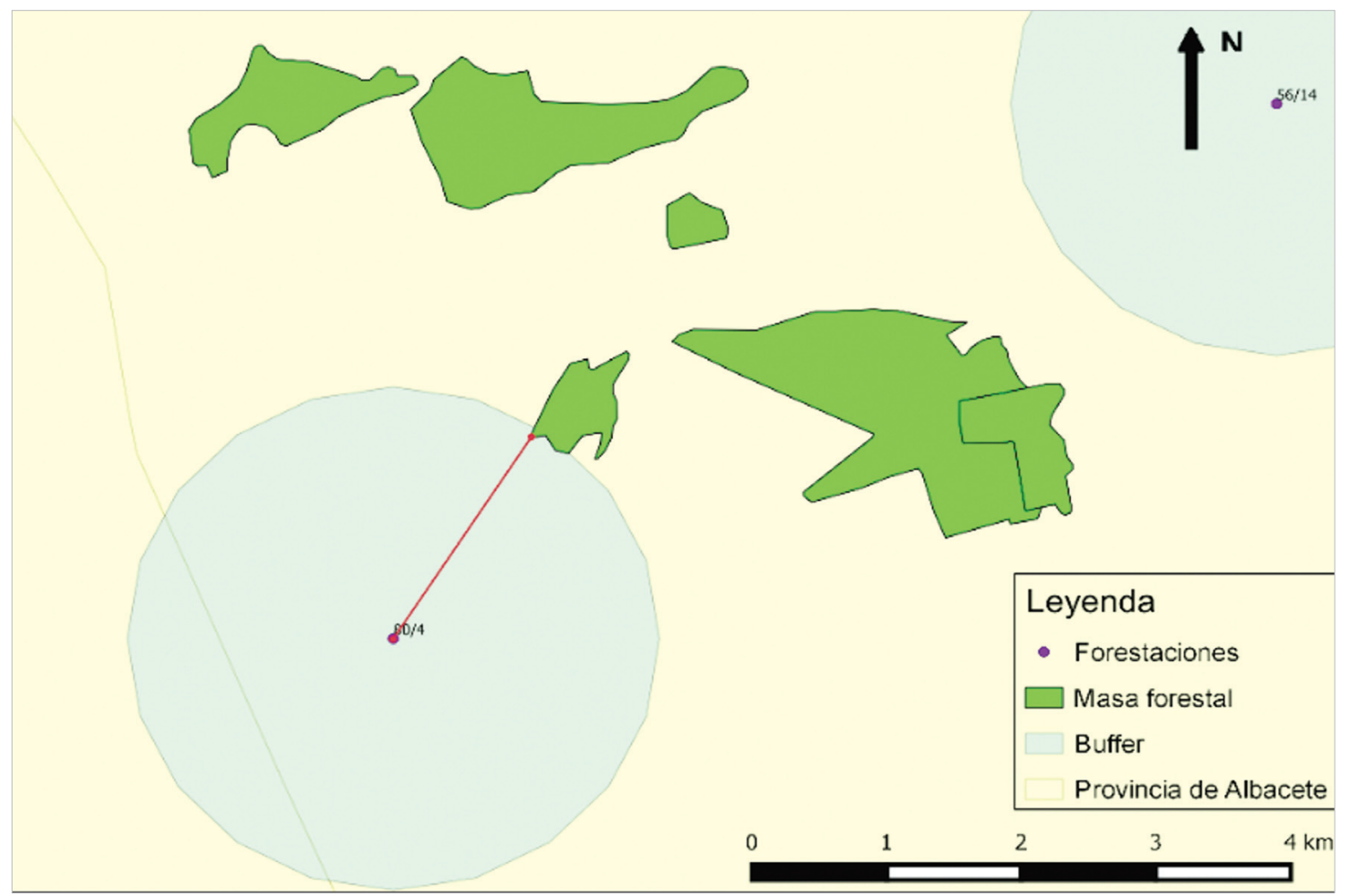

Figura 3. Imagen que ilustra la metodología seguida en la determinación de la distancia de una forestación a una masa forestal. Elaboración propia. 


\subsection{Tratamiento estadístico}

En el tratamiento de los datos se utilizó el paquete estadístico STATGRAPHICS Centurion XVI 16.0.07. Para el estudio de la flora arvense se realizaron diversos análisis estadísticos (ANOVA) con la variable dependiente diversidad (Riqueza específica o Índice de Shannon-Wiener) y los factores edad de la forestación (tres niveles) y posición de la parcela dentro de la forestación (tres niveles). En el análisis, puntualmente, se ha excluido algún valor atípico detectado por el programa estadístico. Para la búsqueda de diferencias significativas entre los niveles de cada factor se ha empleado el Test LSD (Least square difference) de rangos múltiples con una significación del $95 \%$. Cuando se ha relacionado la cobertura de la flora arvense con la cobertura de la forestación, al tratarse de dos variables cuantitativas, se ha utilizado un análisis de regresión lineal simple. Como método de comprobación de la influencia de la distancia a masas forestales sobre la diversidad de flora arvense en las forestaciones, se ha realizado un análisis de regresión lineal múltiple considerando como variable respuesta el número de especies y como variables explicativas continuas, la distancia (tres niveles) y la edad de la forestación (tres niveles).

En todos aquellos casos en los que los datos aparecían registrados como porcentajes con una distribución binomial, se ha realizado previamente su ajuste y transformación por medio del cálculo de la raíz cuadrada de su arcoseno, para ajustar su distribución a la normal.

\section{Resultados y discusión}

\subsection{Caracterización de las forestaciones estudiadas}

Las forestaciones estudiadas presentaron una superficie media de 10,81 ha (mínima, 1,25 ha y máxima, 40,20 ha). La pendiente varió entre el 0,6\% y el 20,9\%, aunque más del $80 \%$ de las forestaciones no alcanzaron el $10 \%$, y la altitud entre los 631,2 y los 971,6 m s.n.m. La altitud media de las forestaciones fue de 811,5 m s.n.m y la pendiente media del $6.3 \%$.

El marco de plantación predominante fue de $4 \times 3 \mathrm{~m}$, apareciendo entre las más antiguas $3 \times 3 \mathrm{~m}$ y entre las más recientes $4 \times 4 \mathrm{~m}$. Las densidades de plantación variaron por tanto desde los 625 pies ha $^{-1}$ hasta los 1111 pies ha $^{-1}$. Como puede observarse, aunque en algunos casos las densidades no fueron muy altas, en comparación con las repoblaciones forestales tradicionales, la gestión forestal sí que puede presentar un problema en el futuro para sus propietarios, por la falta de rentabilidad de muchas de estas forestaciones y, por tanto, para la realización de trabajos selvícolas (Ruiz de la Torre, 1993b).

Las especies arbóreas más utilizadas en las forestaciones estudiadas fueron Pinus halepensis Mill. (pino carrasco) y Quercus ilex subsp. ballota, (Desf.) Samp., pero también se utilizó Pinus pinea L. (pino piñonero) y en algún caso muy con- 
creto Pinus pinaster Ait. Entre las arbustivas se encontraron Retama sphaerocarpa (L.) Boiss., Quercus coccifera L. y Ramnus lycioides L.

La especie dominante en la mayoría de los casos fue el pino carrasco (67\%), siéndolo en un $15 \%$ de las forestaciones la encina y en un $6 \%$ el pino piñonero. En el $12 \%$ restante no había una especie dominante.

Según Ruiz de la Torre (1993b) y Gil y Prada (1993), es indispensable recurrir a la utilización de las diferentes especies de pinos en suelos degradados, como en algunos de los suelos agrícolas utilizados para las forestaciones, dado que reúnen las condiciones necesarias de heliofilia, xerofilia y frugalidad para instalarse y pervivir en dichos terrenos. Se ha de buscar, por tanto, el favorecer a las especies susceptibles de dar paso y buena acogida en el futuro a otras de temperamento más delicado, que puedan alcanzar con el tiempo sistemas de mayor estabilidad y diversidad.

En la misma línea, Gil y Prada (1993) indican que algunas especies de pinos mediterráneos (Pinus pinea, $P$. halepensis, $P$. pinaster, etc.) tienen una morfología adaptada a climas extremos, con gran capacidad de almacenar agua en tallos y hojas y con un rápido cierre de estomas cuando escasea el agua, resultando especies adecuadas para repoblar terrenos pobres que van a padecer prolongadas sequias y temperaturas extremas.

La cobertura de las forestaciones varió desde el 5,14\% en las más jóvenes (17 años) hasta el 54,17\% en las de 15-21 años. Este parámetro está relacionado con la edad, la proporción de cada especie utilizada y la densidad de plantación de la forestación.

Para comprobar la variación de la cobertura de las forestaciones con el paso del tiempo se realizó un ANOVA simple con la variable cobertura de la forestación y el factor edad (tres grupos) y el resultado indicó que existen diferencias significativas entre los tres grupos de edad (F2,35 = 46,50; $p<0,001 ;$ Fig. 4).

Estos datos nos indican que, entre el primer grupo de edad (1-7 años) y el segundo (8-14), es decir en apenas siete años, la cobertura de la forestación aumentó considerablemente, lo que podría haber ocasionado una reducción importante de la llegada de luz al suelo. Así, en el tercer grupo de edad se observa que, debido a la elevada cobertura, si no se realizan tareas selvícolas, la entrada e instalación de nuevas especies (herbáceas y leñosas) tendrán grandes dificultades (Koncz et al., 2011). Algunos autores indican en sus trabajos que la ausencia de la gestión forestal puede ir en detrimento de la diversidad y, por el contrario, la realización de clareos y claras puede tener efectos importantes en la productividad y diversidad del sotobosque (Brockerhoff et al., 2008; Vázquez-Piqué et al., 2009; Navarro et al., 2010).

En todas las forestaciones se detectó en mayor o menor medida, la presencia de plantas herbáceas, pero solo aparecieron especies leñosas en menos de la mitad de ellas (47\%). También es importante destacar que el 73\% de las forestaciones se realizaron sobre cultivos abandonados de cereales o leñosas, mientras que el $27 \%$ restante se instaló sobre eriales o espartizales. Los autores Bremer y Farley (2010) indican que, aunque con la forestación en los terrenos agrícolas abandonados puede 


\section{Cobertura media de las forestaciones por grupo de edad}

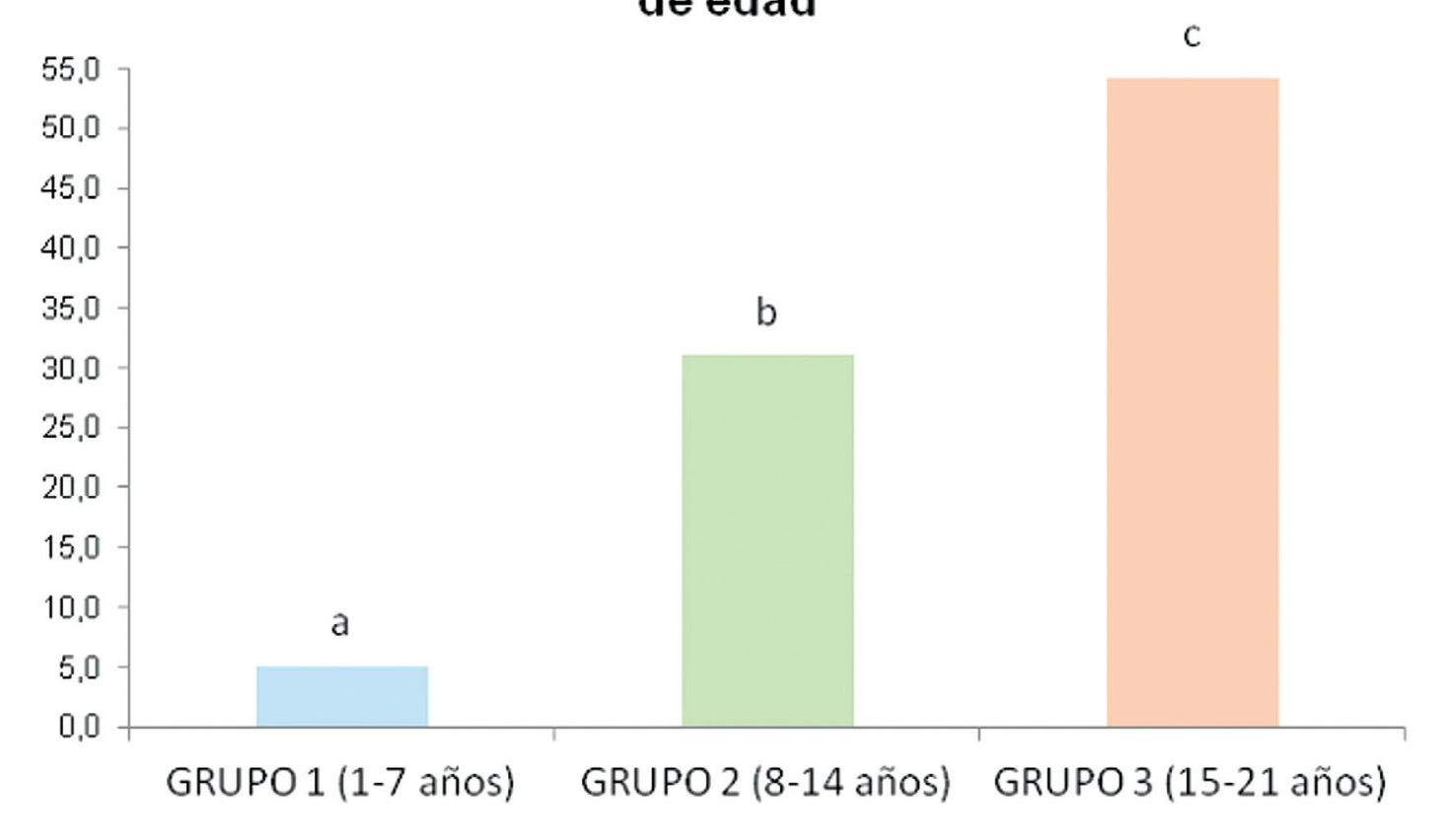

Figura 4. Cobertura media de las forestaciones en función de la edad. Letras diferentes denotan diferencias significativas.

aumentar la diversidad, no ocurre lo mismo cuando se trata de reemplazar la vegetación en terrenos colonizados ya por vegetación forestal. En los resultados obtenidos, no corroborados estadísticamente, se aprecia cierta diferencia en el número de especies arvenses, siendo la riqueza de especies media, aproximadamente de 38 en las parcelas forestadas sobre cultivos, frente a 35 en forestaciones sobre eriales o espartizales.

Entre los antecedentes de las forestaciones estudiadas se puede indicar que, casi en el $90 \%$ de éstas se repusieron las marras, en el $45 \%$ se realizaron, al menos durante el primer año, escardas o laboreos para el control de la vegetación arvense, y que salvo en casos muy aislados no se realizaron clareos, podas o riegos.

\subsection{Flora arvense existente en las forestaciones}

Se contabilizaron 157 especies en la flora aérea. En algunos casos, debido a la dificultad de la identificación de plántulas, no fue posible precisar la especie, y solo se reflejó la familia o el género. Solo en unos pocos casos en los que no se pudo determinar ni siquiera la familia, se consideraron especies sin identificar $(<5 \%)$.

En este sentido Armengot et al. (2011), en un estudio realizado en Cataluña sobre una superficie de muestreo de $196 \mathrm{~m}^{2}$ en cultivos de cereales mediterráneos, obtienen en total 81 especies en la vegetación aérea, cifra inferior a la obtenida en 
este estudio, aunque sobre una superficie muestreada mayor $\left(972 \mathrm{~m}^{2}\right)$. Estos resultados coinciden con los de Bremer y Farley (2010), que señalan como las forestaciones sobre terrenos agrícolas pueden llegar a incrementar su diversidad vegetal. José-María y Sans (2011) destacan, además, la importancia que puede tener el sistema de cultivo previo (ecológico o convencional) sobre la riqueza de especies de flora arvense.

En la flora aérea, los géneros que contaron con un número de especies más elevado fueron Bromus (4), Silene (3), Centaurea (3), Plantago (3), Reseda (3), Euphorbia (3), Crepis (3), Medicago (3), Helianthemum (3) y Teucrium (3). En general y para toda la flora arvense, desde el punto de vista biológico, destacaron principalmente los terófitos como Anacyclus clavatus (Desf.) Pers., Biscutella auriculata L., Bromus rubens L., Centaurea melitensis L., Diplotaxis virgata (Cav.) DC., etc. Apareciendo en menor medida otras formas, los hemicriptófitos como Capsella bursa-pastoris L. y Chondrilla juncea L. o geófitos como Convolvulus arvensis L. y Muscari spp.

En cuanto a la cobertura media de la flora arvense, destacar la diferencia encontrada entre las forestaciones del grupo de mayor edad (20,62\%) frente a las del grupo más joven $(69,76 \%)$. Se observa claramente que este parámetro está directamente influenciado por la cobertura de la forestación, y que disminuye conforme va pasando el tiempo.

Como se puede apreciar en la Figura 5, destaca como principal familia Compositae (Asteraceae) (25\%). Parece lógico si se considera que es una de las familias con mayor diversidad en el mundo. A continuación, aparecen Gramineae y Leguminosae con un $13 \%$ y $9 \%$, respectivamente.

Los porcentajes de representación de estas tres familias, coinciden con otros estudios de comunidades de malas hierbas en terrenos cultivados. En un estudio de comunidades de flora arvense en Andalucía, Saavedra (1987) destaca que las principales familias que aparecen, según el número de géneros y especies, son Compositae, Gramineae y Leguminosae. Hay que recordar, que en estas familias predominan las especies hemicriptófitas, y que estas suelen dominar la flora arvense aérea (Reine y Chocarro, 1993). Estos mismos autores en un trabajo realizado sobre una comunidad herbácea del Pirineo Central, destacan que en la vegetación aérea predominan de una manera importante las gramíneas (68.52\%) frente a las leguminosas $(7.61 \%)$. Es interesante destacar la familia Leguminosae por la importancia que puede representar a la hora de facilitar la instalación de las plantas forestales en estos suelos agrícolas, fijando el nitrógeno y enriqueciendo los suelos. Como familias de interés melífero destacan también Lamiaceae y Cistaceae.

Monteiro et al. (1995) en un estudio sobre flora arvense en cultivos de oleaginosas, obtienen unos datos similares a los de este estudio, destacando la importancia de las familias Compositae (18.2\%), Gramineae (18\%) y Leguminosae (8.5\%) y hasta 30 familias vegetales. Los resultados nos indican que, durante los primeros años de las forestaciones, existe una gran diversidad de plantas como lo demuestra la presencia de hasta 30 familias, dato que coincide con el del último trabajo citado (Monteiro et al., 1995), que incluyen herbáceas y leñosas y que van apareciendo progresivamente con el paso del tiempo. 


\section{Principales familias en la flora arvense (\%)}

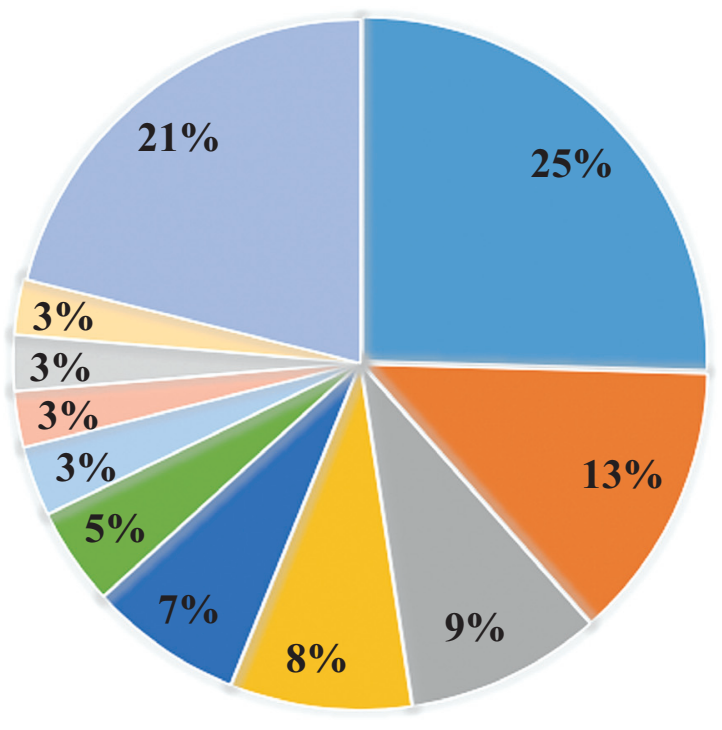

\begin{tabular}{l|l}
$\square$ Compositae & $\begin{array}{l}\text { Euphorbiaceae } \\
\text { Orobanchaceae } \\
\text { Resedaceae } \\
\text { Gramineae } \\
\text { Rubiaceae } \\
\text { Campanulaceae } \\
\text { Caprifoliaceae } \\
\text { Geraniaceae } \\
\text { Lamiaceae } \\
\text { Cruciferae } \\
\square \text { Caryophyllaceae } \\
\square \text { Cistaceae } \\
\text { Apiaceae } \\
\text { Boraginaceae } \\
\text { Plantaginaceae } \\
\text { Chenopodiaceae } \\
\text { Convolvulaceae } \\
\text { Hypericaceae } \\
\text { Papaveraceae } \\
\text { Plumbaginaceae } \\
\text { Polygalaceae } \\
\text { Rosaceae } \\
\text { Scrophulariaceae } \\
\text { Thymelaeaceae } \\
\text { Valerianaceae } \\
\text { Xanthorrhoeaceae }\end{array}$ \\
\hline
\end{tabular}

Figura 5. Principales familias encontradas en la flora arvense en las 36 forestaciones estudiadas (\%).

\subsection{Evolución de la flora arvense con la edad de las forestaciones}

Los resultados mostraron un mayor número de especies de flora arvense en las forestaciones más jóvenes, con una media aproximada de 27, frente a 20 y 21 en los siguientes dos grupos de edad, respectivamente. En cualquier caso, hay que tener en cuenta que la Riqueza específica es un índice muy relacionado con el tamaño de la muestra (Escolástico et al., 2006), y en las forestaciones estudiadas aún con grandes diferencias de superficies, se ha muestreado el mismo número de parcelas para todas ellas. Este índice, según algunos autores, se debe de considerar más bien como índice de riqueza y no de diversidad y tiene sus limitaciones a la hora de hacer comparaciones, ya que nos obliga a contabilizar todas las especies de una comunidad (Hurlbert, 1971).

En el análisis estadístico realizado (ANOVA multifactorial) se ha utilizado como variable la riqueza específica y como factores la edad (tres grupos) y la posición de la parcela (tres grupos). Los resultados nos indican que existen diferencias significativas entre el primer grupo de edad y los otros $\operatorname{dos}\left(\mathrm{F}_{2,107}=11,52 ; \mathrm{p}<\right.$ $0,001)$, y entre las tres posiciones de las parcelas $\left(\mathrm{F}_{2,107}=17,41 ; \mathrm{p}<0,001\right)$. La riqueza específica disminuye con el paso del tiempo y desde la parcela exterior hacia la más interior (Tabla 1).

Para comprobar si existe una relación de dependencia entre el número de especies de flora arvense y la cobertura de la forestación se ha realizado un análisis de 
regresión lineal simple, encontrándose un modelo estadísticamente significativo $\left(\mathrm{F}_{1,35}=7,90 ; \mathrm{p}=0,0081\right)$ con la siguiente ecuación de regresión: $\mathrm{N}^{\mathrm{o}}$ Especies Totales $=44,803-14,746$ x Cobertura de la Forestación $(\%)$ y una $\mathrm{R}^{2}=18,87 \%$. Se observa que el número de especies de flora arvense disminuye con la cobertura de la forestación y, en este caso, el modelo de regresión ajustado explicaría algo menos del 19\% de la variación total de la variable dependiente.

Los resultados sobre la flora arvense arrojaron un número medio de especies por forestación de 23 , sobre una superficie muestreada de $27 \mathrm{~m}^{2}$, aunque cabe destacar que en algún caso concreto se llegaron a alcanzar hasta 43 especies. Trabaud et al. (1997) encuentran 24 especies en suelos forestales con una superficie de muestreo de $100 \mathrm{~m}^{2}$, una cifra proporcionalmente menor a la obtenida en este trabajo. Sin embargo, Reine y Chocarro (1993), sobre una superficie de muestreo de $2,25 \mathrm{~m}^{2}$, obtienen 34 especies en una comunidad pratense.

Los datos revelan que la cobertura del suelo por la flora arvense fue mayor en las forestaciones de menor edad, con una media de $69,76 \%$ de cobertura del suelo, frente a $33,17 \%$ y $20,62 \%$ en los dos siguientes grupos de edad, habiéndose observado, en este caso, que en la parcela $\mathrm{A}$, por el efecto borde, las diferencias son también importantes $75,60 \%$ frente a $46,01 \%$ y $29,03 \%$, respectivamente. Esto indica que, en los primeros momentos de la forestación, la superficie del suelo está cubierta por la flora arvense y, por tanto, existe una mayor competencia con las plantas recién instaladas, aunque al mismo tiempo dicha vegetación ayuda a una mayor protección del suelo. Por otra parte, es lógico que las parcelas del borde de la forestación tengan más facilidad para reclutar nuevas especies, debido fundamentalmente a una mayor luminosidad.

Los resultados del análisis estadístico (ANOVA multifactorial), utilizando como variable la cobertura de flora arvense y como factores la edad (tres grupos) y la posición de la parcela (tres grupos), nos indican que existen diferencias significativas entre los diferentes grupos de edad $\left(\mathrm{F}_{2,107}=56,04 ; \mathrm{p}<0,001\right)$ y entre la posición más externa y las interiores $\left(\mathrm{F}_{2,107}=6,67 ; \mathrm{p}=0,0019\right)$. Como en el caso de la Riqueza específica también se detecta en este caso una disminución de la cobertura de la flora arvense con el paso del tiempo y hacia el interior de la forestación (Fig. 6).

Se realizó también un análisis de regresión lineal simple para comprobar la relación de dependencia que existe entre la cobertura de las forestaciones y la cobertura de la flora arvense, resultando un modelo lineal simple estadísticamente significativo $\left(\mathrm{F}_{1,35}=28,98 ; \mathrm{p}<0,001\right)$, con la expresión: Cobertura de Flora $(\%)=$ $0,637-0,228$ x Cobertura de la Forestación (\%) y una $\mathrm{R}^{2}=46,02 \%$. De esta manera, el modelo de regresión ajustado explicaría algo más del $46 \%$ de la variación total de la variable dependiente.

Con respecto al índice de diversidad de Shannon, se observa una tendencia a disminuir desde las forestaciones recién implantadas hasta aquellas que están instaladas desde hace tiempo (15-21 años), con valores de 2,45 para forestaciones jóvenes frente a 2,22 para las más viejas disponibles. De la misma manera si no tenemos en cuenta la parcela situada al borde de la forestación las diferencias aumentan desde 2,39 en las forestaciones más jóvenes hasta 2,09 en las más viejas. 


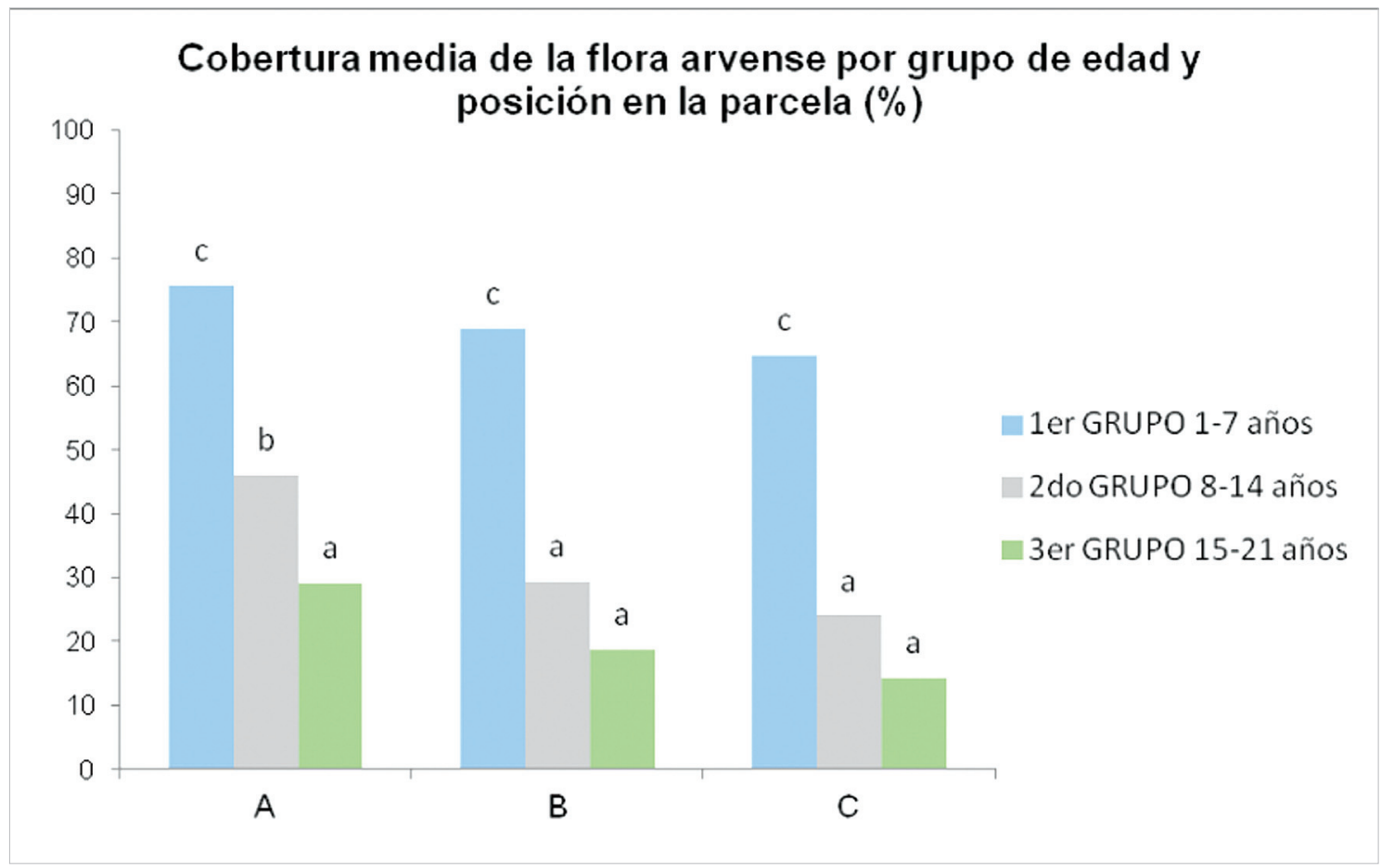

Figura 6. Cobertura media de la flora arvense (\%) según grupo de edad y posición de la parcela (A, $\mathrm{B}$ y C) en la forestación. Letras diferentes denotan diferencias significativas.

Se realizó un ANOVA multifactorial con la variable Índice de Shannon y los factores edad de la forestación (tres grupos) y posición de la parcela (tres grupos). Se han eliminado algunos valores incoherentes que por su magnitud obedecían a errores de medición o de toma de datos y el resultado nos indica que existen diferencias significativas entre el primer grupo de edad y los otros dos $\left(\mathrm{F}_{2,104}=4,81 ; \mathrm{p}\right.$ $=0,0035)$ y entre la posición de la parcela más externa y la demás $\left(\mathrm{F}_{2,104}=5,99 ; \mathrm{p}\right.$ $=0,0101$; Fig. 7).

Con estos resultados se observa que la diversidad de las especies arvenses disminuye con el paso del tiempo y desde la parcela más externa a la más interna. Esto se puede explicar por la mayor cobertura de la vegetación forestal con el paso del tiempo y desde el borde de la parcela al interior de la forestación, evitando el paso de la luz y compitiendo de una forma más importante que en los primeros años desde la plantación.

El índice de Shannon es sobre todo un índice informativo, que tiene en cuenta además del número de especies presentes en la comunidad, cómo se distribuye la abundancia entre las diferentes especies, dando igual peso a todas ellas. Este índice nos informa, por tanto, de la riqueza de especies y la equitabilidad en un solo valor (Escolástico et al., 2006), lo que nos ha permitido comparar la diversidad de las diferentes comunidades estudiadas.

En la Tabla 1 se muestra un resumen de los diferentes Índices de diversidad cal- 


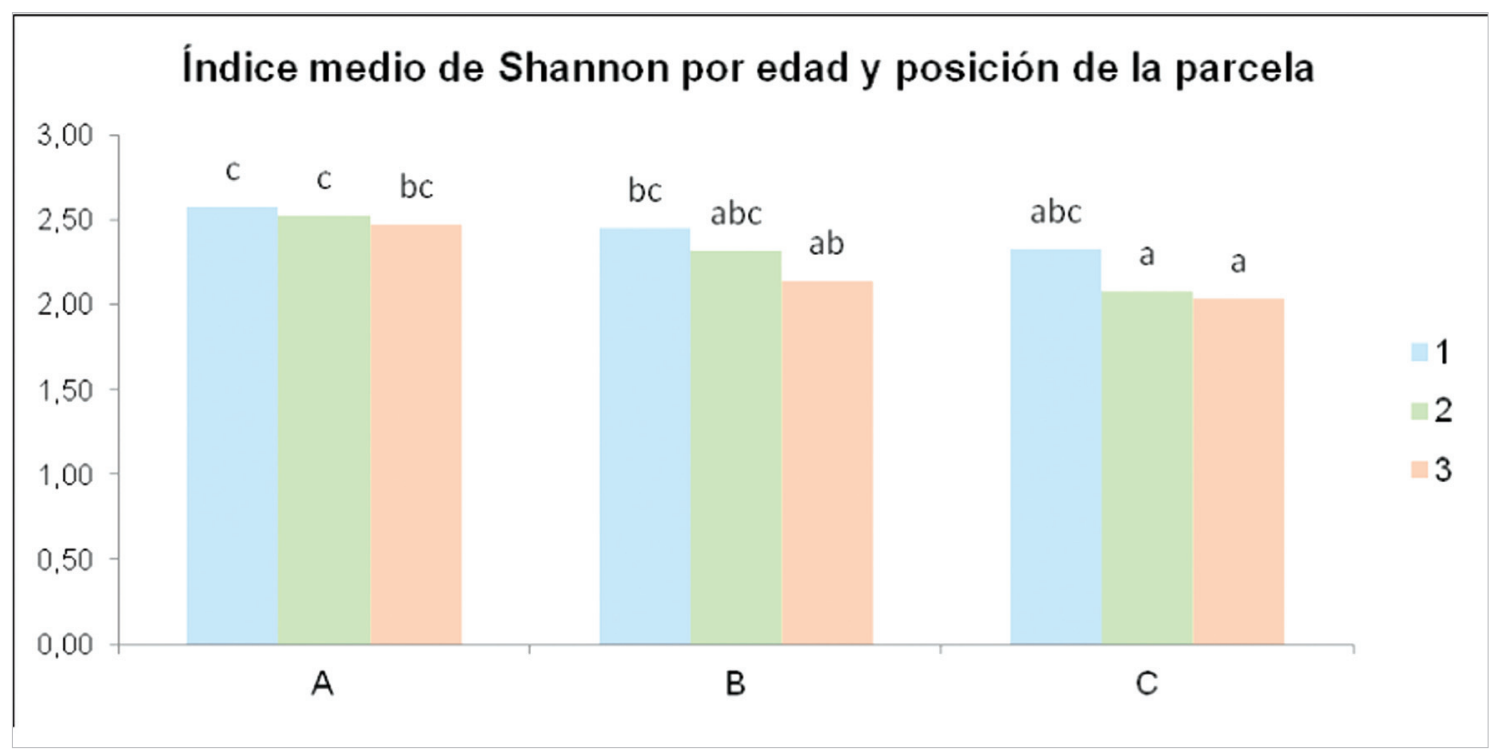

Figura 7. Índice medio de Shannon según edad de la forestación (Grupos 1, 2 y 3) y posición de la parcela (A, B y C) en la forestación. Letras diferentes denotan diferencias significativas.

culados para las comunidades de flora arvense estudiadas, en los que se comprueba, como se ha indicado anteriormente, que existe una mayor diversidad en los primeros años de instalación de la forestación y en las parcelas exteriores.

Tabla 1. Resumen de los diferentes Índices de diversidad calculados (media \pm desviación estándar) en función de la edad y de la posición de la parcela en la forestación.

\begin{tabular}{ccccccccccc}
\hline Índices & & $\begin{array}{c}\text { GRUPO 1 } \\
(1-7 \text { años })\end{array}$ & & \multicolumn{3}{c}{$\begin{array}{c}\text { GRUPO 2 } \\
(8-14 \text { años })\end{array}$} & \multicolumn{4}{c}{$\begin{array}{c}\text { GRUPO 3 } \\
(15-21 \text { años })\end{array}$} \\
\hline & $\mathrm{A}$ & $\mathrm{B}$ & $\mathrm{C}$ & $\mathrm{A}$ & $\mathrm{B}$ & $\mathrm{C}$ & $\mathrm{A}$ & $\mathrm{B}$ & $\mathrm{C}$ \\
\hline Riqueza & 30 & 26 & 24 & 24 & 20 & 17 & 27 & 20 & 16 \\
específica & $\pm 6,47$ & $\pm 6,07$ & $\pm 5,66$ & $\pm 5,53$ & $\pm 5,01$ & $\pm 3,96$ & $\pm 5,02$ & $\pm 6,61$ & $\pm 7,69$ \\
\hline \multirow{2}{*}{ Shannon } & 2,58 & 2,45 & 2,33 & 2,52 & 2,31 & 2,07 & 2,47 & 2,14 & 2,04 \\
& $\pm 0,46$ & $\pm 0,51$ & $\pm 0,49$ & $\pm 0,32$ & $\pm 0,37$ & $\pm 0,49$ & $\pm 0,50$ & $\pm 0,40$ & $\pm 0,33$ \\
\hline
\end{tabular}

A: borde; B: a $25 \mathrm{~m}$ del borde hacia el interior; C: a $50 \mathrm{~m}$ del borde hacia el interior.

Es importante destacar que, aunque la diversidad de la flora arvense disminuye con el paso del tiempo, nos encontramos ante estados más evolucionados de vegetación y, por tanto, con posibilidades de mejorar las condiciones edáficas y microclimáticas que pueden ayudar a alcanzar etapas más altas en la sucesión vegetal. Para Ruiz de la Torre (1993b) la diversidad biológica crece con el grado de madurez, y las diferentes etapas evolutivas se miden por el incremento de los índices de diversidad, pero al mismo tiempo considera también que aumentar la diversidad es favorecer el incremento de la madurez y su mantenimiento el máximo tiempo posible. 


\subsection{Estudio de las especies leñosas}

Algunos trabajos han puesto de manifiesto desde hace tiempo el efecto facilitador de los matorrales en la restauración de zonas degradadas del bosque mediterráneo (Villa et al., 2012; Costa et al., 2017). Dichos autores obtuvieron mejores resultados en una plantación de encina y rebollo bajo Cytisus multiflorus (L'Hér.) Sweet. que, en suelo desnudo, por eso parece importante conocer el proceso de colonización y las especies de leñosas que van instalándose en forestaciones de terrenos cultivados abandonados.

Como se ha indicado antes, las leñosas han aparecido en un $47 \%$ de las forestaciones estudiadas, destacando algunas especies de los géneros Artemisia, Helianthemum, Helichrysum, Teucrium y Thymus entre otros. El número de especies leñosas encontradas en este estudio, en las diferentes forestaciones, alcanza la cifra de 24 en el conjunto de los diferentes grupos de edad.

En lo referente al porcentaje de leñosas aparecidas en las diferentes forestaciones por edad (tres grupos) y posición (A, B y C), los resultados revelan que las leñosas fueron apareciendo con el paso del tiempo, un $4 \%$ en las forestaciones más jóvenes y $10 \%$ y $12 \%$ en las forestaciones de $8-14$ y 15-21 años, respectivamente. Esta diferencia no se observa, sin embargo, según la posición de la parcela en la forestación. Al realizar el análisis estadístico (ANOVA Multifactorial), se comprobó que, aunque para la posición de la parcela no existieron diferencias significativas $\left(\mathrm{F}_{2,107}=0,27 ; \mathrm{p}>0,05\right)$, sí que se observaron entre el primer grupo de edad y los demás $\left(F_{2,107}=5,06 ; p=0,008\right)$, lo que nos indica que a partir de los 8-14 años se incrementa la presencia de leñosas (Tabla 2).

Tabla 2. Número de especies leñosas (\%) en función de la edad y de la posición de la parcela en la forestación.

\begin{tabular}{cccccccccc}
\hline \multicolumn{8}{c}{$\mathbf{N}^{\mathbf{0}}$ de especies leñosas (\%) según la edad y posición en la forestación } \\
\hline $\mathrm{N}^{0}$ & \multicolumn{3}{c}{$\begin{array}{c}\mathrm{1}^{\text {er }} \text { GRUPO } \\
(1-7 \text { años })\end{array}$} & & & $\begin{array}{c}2^{0} \text { GRUPO } \\
(8-14 \text { años })\end{array}$ & & \multicolumn{3}{c}{$\begin{array}{c}3^{\text {er }} \text { GRUPO } \\
(15-21 \text { años })\end{array}$} \\
\hline Media & 6,94 & 1,99 & 3,08 & 8,95 & 10,53 & 10,40 & 12,27 & 13,82 & 9,15 \\
\hline $\begin{array}{c}\text { Desviación } \\
\text { estándar }\end{array}$ & 6,51 & 2,86 & 3,42 & 12,68 & 14,10 & 12,56 & 11,65 & 12,67 & 12,10 \\
\hline
\end{tabular}

A: borde; B: a $25 \mathrm{~m}$ del borde hacia el interior; $\mathbf{C}$ : a $50 \mathrm{~m}$ del borde hacia el interior.

\subsection{Relación entre la colonización de plantas y la distancia a masas forestales}

Como ya se indicó en el apartado de metodología, se calcularon las distancias de las forestaciones a las masas forestales más cercanas con el objeto de comprobar si existe relación entre estas y la velocidad de colonización de especies leño- 
sas. Para comprobar esta relación de dependencia, se realizó un análisis de regresión lineal con la variable respuesta número de especies leñosas presentes en la flora aérea.

Los resultados nos indican que existe un modelo lineal estadísticamente significativo que relaciona la presencia de leñosas, la edad de la forestación y la distancia a masas forestales en la flora arvense $\left(\mathrm{F}_{2,35}=5,38 ; \mathrm{p}=0,0095\right)$. Dicho modelo tiene por expresión la ecuación: Leñosas $(\%)=0,174+0,009 \times$ Edad - 0,043 x Distancia, con una $\mathrm{R}^{2}=24,59 \%$, observándose una mayor presencia de especies leñosas en la flora aérea cuando la distancia a masas forestales es menor, de esta manera, el modelo de regresión ajustado explica casi un $25 \%$ de la variación total de la variable dependiente.

Estos resultados coinciden con otros trabajos sobre la restauración natural de terrenos cultivados abandonados, en los que se demuestra la importancia del paso del tiempo en la incorporación de especies leñosas (Sánchez, 1995). Por otra parte, Zapata et al. (2013) indican que, aunque tiene una gran importancia la forma de dispersión de las semillas (viento, animales, escorrentía, etc.), en algunos casos una forestación situada lejos de una masa forestal puede, sin embargo, recibir aporte de semillas por los animales (aves) que de otro modo no llegarían.

\section{Conclusiones}

Los resultados permiten confirmar que, si no se realizan tareas selvícolas, las forestaciones con altas densidades alcanzan con el tiempo valores de cobertura muy elevados que pueden reducir considerablemente la llegada de luz al suelo, y dificultar la entrada e instalación de nuevas especies (herbáceas y leñosas). Por otra parte, la cobertura de la flora arvense disminuye con el paso del tiempo, desde el borde hacia el interior, y a medida que aumenta la cobertura de la forestación.

La diversidad de la vegetación herbácea también disminuye con el tiempo y desde la parcela más externa a la más interna, mientras que las especies leñosas se van incorporando lentamente y no se observan diferencias significativas entre el exterior y el interior de la forestación.

La importancia del paso del tiempo y la distancia a masas forestales están muy relacionadas con la introducción e instalación de especies típicas de estadíos sucesionales más avanzados.

\section{Bibliografía}

Armengot, L.; José-María, L.; Blanco-Moreno, J.M.; Romero-Puente, A.; Sans, F.X.; 2011. Landscape and land-use effects on weed flora in mediterranean cereal fields. Agriculture, ecosystems and environment. 142 (3-4), 311-317. https://doi.org/10.1016/ j.agee.2011.06.001

Boccanelli, S.I.; Pire, E.F.; Torres, P.S.; Lewis, J.P.; 1999. Cambios en la vegetación de un 
campo abandonado después de un cultivo de trigo. Pesquisa Agropecuaria Brasileira. 34 (2), 151-157. https://doi.org/10.1590/S0100-204X1999000200001

Bremer, L.L.; Farley, K.A.; 2010. Does plantation forestry restore biodiversity or create green deserts? A synthesis of the effects of land-use transitions on plant species richness. Biodivers. Conser. 19, 3893-3915. https://doi.org/10.1007/s10531-010-9936-4

Brockerhoff, E.G.; Jactel, H.; Parrotta, J.A.; Quine, C.P.; Sayer, J.; 2008. Plantation forest and biodiversity: oximoron or opportunity? Biodivers. Conser. 17, 925-951. https://doi. org/10.1007/s10531-008-9380-x

Carmona, J.; Orozco, E.; Monreal, J.A.; Selva, M.; 2007. Experiencias sobre el control de malas hierbas en las forestaciones realizadas en la provincia de Albacete durante los últimos años (2002-2006). In: Mansilla, J.; Artigao, A.; Monreal, J.A.; (eds.), La malherbología en los nuevos sistemas de producción agraria. XI congreso de la sociedad española de malherbología, 239-242.

Carmona, J.; 2008. Aspectos medioambientales del programa de forestación de tierras agrícolas en la provincial de Albacete. Medio ambiente. Castilla-La Mancha. 19, 30-33.

Chapman, C.A.; Chapman, L.J.; 1999. Forest restoration in abandoned agricultural land: A case study from East Africa. Conservation Biology, 13 (6), 1301-1311. https://doi.org/ 10.1046/j.1523-1739.1999.98229.x

Costa, A.; Villa, S.; Alonso, P.; García-Rodríguez, J.A.; Martín, F.J.; Martínez-Ruiz, C.; Fernández-Santos, B.; 2017. Can native shrubs facilitate the early establishment of contrasted co-occurring oaks in Mediterranean grazed areas? J Veg. Sci. 28, 1047-1056. https: // doi.org/10.1111/jvs.12550

Díaz, J.L.; 1997. Aspectos diferenciales de los suelos agrícolas y forestales para la forestación. In: Orozco, E.; Monreal, J.A.; (eds.), Forestación en tierras agrícolas. 43-71. UC LM. Cuenca.

Escolástico, C.; Cabildo, M.P.; Claramunt, R.M.; Claramunt, T.; 2006. Ecología II: Comunidades y ecosistemas. Universidad Nacional de Educación a Distancia, Madrid.

Francis, C.F.; 1990. Variaciones sucesionales y estacionales de vegetación en campos abandonados de la provincial de Murcia. Ecología, 4, 35-47.

Gil, L.; Prada, A.; 1993. Los pinos como especies básicas de la restauración forestal en el medio mediterráneo. Ecología. 7, 113-125.

Hurlbert, S.H.; 1971. The nonconcept of species diversity: a critique and alternative parameters. Ecology. 52 (4), 577-586. https://doi.org/10.2307/1934145

Koncz, G.; Török, P.; Papp, M.; Matus, G.; Tóthmérész, B.; 2011. Penetration of weeds into the herbaceous understorey and soil seed bank of a turkey oak-sessile oak forest in Hungary. Community ecology. 12 (2), 227-233. https://doi.org/10.1556/ComEc.12.20 11.2.11

Kushla, J.D.; 2009. Afforestation in North Mississippi on retired farmland using Pinus echinata: First-year results. Southern Journal of Applied Forestry, 33 (3), 142-144.

Lemieux, C.; Delisle, C.; 1998. Using cover crops to establish white and black spruce on abandoned agricultural lands. Phytoprotection, 79 (1), 21-33. https://doi.org/10.7202/ $706132 \mathrm{ar}$

M.A.P.A. 2006. Forestación de tierras agrícolas: Análisis de su evolución y contribución a la fijación del carbono y al uso racional de la tierra. Dirección General de Desarrollo Rural. M.A.P.A.

M.A.P.A.M.A. 2017. Anuario de estadística forestal 2013. Secretaría General Técnica. MA 
PAMA, Madrid.

Monsalve, M.; (coord.). 1997. Manual de forestación. Junta de Castilla y León. Salamanca. Monteiro, A.; Figueira, T.; Vasconcelos, T.; Moreira, I.; 1995. Sunflower weed flora in Portugal. Actas del Congreso de la Sociedad Española de Malherbología. 87-90.

Montiel, C.; Galiana, L.; 2004. La restauración de paisajes forestales a través de la forestación de tierras agrarias. Cuad. Soc. Esp. Cien. For, 17, 193-198.

Montoya, J.M.; 1997. El Real Decreto 378/93 y la problemática de su aplicación por el profesional forestal. In: E. Orozco y J. A. Monreal (eds.), Forestación en tierras agrícolas. 183-197. UCLM. Cuenca.

Navarro, F.B.; Jiménez, M.N.; Cañadas, E.M.; Gallego, E.; Terrón, L.; Ripoll, M.A.; 2010. Effects of different intensities of overstory thinning on tree growth and understory plant -species productivity in a semi-arid Pinus halepensis mill. afforestation. Forest Systems. 19 (3), 410-417. https://doi.org/10.5424/fs/2010193-8858

Orozco, E.; Lopez, F.R.; (coord.). 1993. Selvicultura Mediterránea. UCLM. Cuenca.

Orozco, E.; Monreal, J.A.; (coord.). 1997. Forestación en tierras agrícolas. UCLM, Cuenca.

Pires, A.L.; Xavier, R.; 2010. Influence of vegetation management and fertilization on Pinus pinaster growth and on understory biomass and composition. Forest Systems, 19 (3), 404-409. https://doi.org/10.5424/fs/2010193-8927

Reine, R.; Chocarro, C.; 1993. Relación entre el banco de semillas del suelo y la vegetación aérea en una comunidad pretense del pirineo central. Pastos. XXIII (I), 89-100.

Ruiz de la Torre, J.; 1990a. Distribución y características de las masas forestales españolas. Ecología, 1, 11-30.

Ruiz de la Torre, J.; 1990b. Mapa forestal de España. Albacete. Hoja 6-8. Instituto Nacional para la Conservación de la Naturaleza. Madrid.

Ruiz de la Torre, J.; 1993a. Mapa forestal de España. Onteniente. Hoja 7-8. Instituto Nacional para la Conservación de la Naturaleza. Madrid.

Ruiz de la Torre, J.; 1993b. Objetivos de diversidad biológica en la reforestación de tierras agrícolas. Elección de especie y densidad de implantación. Montes. (34), 20-30.

Saavedra, M.; 1987. Estudio de las comunidades de flora arvense (malas hierbas) en el valle medio del Guadalquivir. Tesis Doctoral. Universidad de Córdoba.

Sánchez, M.D.; 1995. La reconstrucción natural de la vegetación leñosa en zonas agrícolas abandonadas. Instituto de Estudios Albacetenses, Albacete.

Sánchez, P.; Güemes, J.; Herranz, J.M.; Fernández, S.; López, G.; Martínez, J.J.; 1997. Plantas vasculares endémicas, amenazadas o raras de la provincia de Albacete. Instituto de Estudios Albacetenses, Albacete.

Terradas, J.; 2001. Ecología de la vegetación. Omega, Barcelona.

Trabaud, L.; Martínez, J.J.; Ferrandis, P.; González, A.I.; Herranz, J.M.; 1997. Végétation épigée et banque de semences du sol: leur contribution à la stabilité cyclique des pinèdes mixtes de Pinus halepensis et P. pinaster. Canadian journal of botany. 75, $1012-$ 1021. https://doi.org/10.1139/b97-112

Van Lerberghe, P.; Balleux, P.; 2001. Boisement des terres agricoles. Institut pour le développement forestier, Paris.

Vázquez-Piqué, J.; Salguero, M.L.; González-Pérez, A.; García-Güemes, C.; Tapias, R.; Macías, R.; Allué, C.; 2009. Influencia de las claras de Pinus sylvestris 1. y Pinus nigra Arn. en el crecimiento diametral del alcornoque (Quercus suber) en Bozo (Burgos). Ac- 
tas del V Congreso Forestal Español. 2-13.

Villa, S.; Martínez-Ruiz, C.; García, J.A.; Fernández-Santos, B.; 2012. Influencia de Cytisus multiflores en la supervivencia de plantas de Quercus ilex y Q. pyrenaica durante los dos primeros años. In: Martínez, C.; Lario, F.J.; Fernández, B.; Avances en la restauración de sistemas forestales. 75-80.

Zapata, V.M.; Robledano, F.; Romero, A.; Belmonte, F.; Martínez, V.; Martínez, C.; 2013. Condicionantes ambientales para la reforestación natural de cultivos de secano abandonados en el sureste de la península ibérica. Actas del VI Congreso Forestal Español. 213. 
